\title{
Microscopic Equations of State, Thermodynamical Properties, and System Collapse: Application of the Dynamical Equations of Period Vectors in Crystal Structure Prediction under External Temperature and Stress/Pressure (Version 3)
}

\author{
Gang Liu* \\ ORCID:0000-0003-1575-9290, HPCVL, Queens University, Kingston, Canada
}

(Dated: Jan. 20, 2019)

\begin{abstract}
In crystal structure prediction, Newton's second law can always be applied to particles (atoms or ions) in a cell to determine their positions. However the values of the period vectors (crystal cell edge vectors) should be determined as well. Here we applied the dynamical equations of period vectors derived recently based on Newton's laws (doi:/10.1139/cjp-2014-0518) for that purpose, where the period vectors are driven by the imbalance between the internal and external pressures/stresses. For equilibrium states, they became equations of state, which essentially turn out the equilibrium conditions of crystals from mechanical point of view. Additionally for external pressure, they became Mie-Gruneisen equation supposing the Gruneisen constant is $1 / 3$, which means phonon frequency is inversely proportional to the cell length. Since the internal stress has both a full kinetic energy term and a full interaction term, the influences of both external temperature and stress/pressure on crystal structures can be calculated, then thermodynamical properties and processes were presented. Contrary to usual ideas, the equations show that pure harmonic vibrating phonons can result in thermal expansion in crystals when the external temperature is changed. Finally, crystal system collapse due to temperature and/or stress/pressure change was discussed.
\end{abstract}

\section{INTRODUCTION}

Crystal structure prediction from being questioned becomes more and more feasible and important these years[1-6]. Actually minimizing the enthalpy $H=E+P V$ is normally employed to determine crystal structures for constant external pressure $P$, where $E$ and $V$ are the total potential energy and volume of a crystal cell respectively. Then, one has

$$
\begin{array}{ll}
\frac{\partial(E+P V)}{\partial \mathbf{r}_{i}}=0 & (i=1,2, \cdots, n), \\
\frac{\partial(E+P V)}{\partial \mathbf{h}}=0 & (\mathbf{h}=\mathbf{a}, \mathbf{b}, \mathbf{c}),
\end{array}
$$

where $n$ is the total number of particles (atoms or ions) in a cell, $\mathbf{r}_{i}$ is the position vector of particle $i$, and $\mathbf{h}$ is any one of the period vectors (cell edge vectors) $\mathbf{a}, \mathbf{b}$, and $\mathbf{c}$. Eq.(1) means

$$
\mathbf{F}_{i}=-\frac{\partial E}{\partial \mathbf{r}_{i}}=0 \quad(i=1,2, \cdots, n),
$$

where $\mathbf{F}_{i}$ is the net force acting on particle $i$. Eq.(2) means

$$
\frac{\partial E}{\partial \mathbf{h}}+P \sigma_{h}=0 \quad(\mathbf{h}=\mathbf{a}, \mathbf{b}, \mathbf{c}),
$$

where $\sigma_{h}=\partial V / \partial \mathbf{h}$ is the cell surface vector with respect to $\mathbf{h}$. Actually the way in our previous paper[7] is equivalent to this. However the temperature effect is not shown there.

Another way making much progress in crystal structure prediction is minimizing Gibbs free energy and applying quasiharmonic Debye model[8-14] for phonon (then temperature) effect[15-19]. However, they usually use the cell volume as variable rather than the period vectors. Additionally all above only consider the external pressure, rather than the general external stress.

In this paper, we will apply the dynamical equations of period vectors derived recently[20] in crystal structure prediction, with temperature including phonon effect applied, and external stress considered. As a result, equations

*Electronic address: gang.liu@queensu.ca,gl.cell@outlook.com 
of state, equivalent to the equilibrium conditions of crystals from mechanical point of view, is derived. For external pressure, they became Mie-Gruneisen equation supposing the Gruneisen constant is $1 / 3$, which means phonon frequency is inversely proportional to the cell length. Contrary to usual ideas, the equations also show that pure harmonic vibrating phonons can result in thermal expansion in crystals when the external temperature is changed. Later, crystal system collapse due to temperature and/or stress/pressure change is discussed.

\section{DYNAMICAL EQUATIONS OF PERIOD VECTORS IN CRYSTAL STRUCTURE}

In our recent paper[20], particles are always obeying Newton's second law

$$
m_{i} \ddot{\mathbf{r}}_{i}=\mathbf{F}_{i} \quad(i=1,2, \cdots, n) .
$$

When the system reaches an equilibrium state, Eq.(5) becomes Eq.(3). For determing the period vectors, the dynamical equations of them were derived based on Newton's laws as[20]

$$
\alpha_{\mathbf{h}, \mathbf{h}} \ddot{\mathbf{h}}=(\pi+\mathbf{\Upsilon}) \cdot \sigma_{h} \quad(\mathbf{h}=\mathbf{a}, \mathbf{b}, \mathbf{c}),
$$

where $\alpha_{\mathbf{h}, \mathbf{h}}$ is some mass, tensor $\pi$ is the internal stress, and tensor $\boldsymbol{\Upsilon}$ is the external stress. As it shows, the imbalance between the internal and external stresses drive the period vectors to change. As a matter of fact, crystal structure normally means the values of particle positon vectors and the period vectors in an equilibrium state, then let us regard all the so-called degrees of freedom (DOF) of the system in the paper[20], which are $\mathbf{r}_{1}, \mathbf{r}_{2}, \cdots, \mathbf{r}_{n}, \mathbf{a}, \mathbf{b}$, and $\mathbf{c}$, as those in the equilibrium state. Since the purpose of pursuing a crystal structure is to determine their final values from some guessed ones, the velocities of them can be completely forgotten. Then let us ignore the left side of Eq.(6), but only change the period vectors in the directions of the calculated result of the right side of Eq.(6).

The internal stress in Eq.(6) has two terms

$$
\pi=\tau+\varepsilon
$$

The first tensor term

$$
\tau=\frac{1}{3 V} \sum_{i=1}^{n} m_{i}\left|\dot{\mathbf{r}}_{i}\right|^{2} \mathbf{I}=\frac{2}{3 V} E_{k, M D} \mathbf{I},
$$

where $E_{k, M D}$ is the total kinetic energy of the particles in the center cell and $\mathbf{I}$ is an identity tensor, then this term should be temperature dependent. Although no kinetic energy for the DOF variables, which are the crystal framework (backbone) in equilibrium states, other kinetic energy do exist in the system separately. For example, the actual position vector of particle $i$ is $\mathbf{r}_{i}+\Delta \mathbf{r}_{i, v b}+\Delta \mathbf{r}_{i, t h}$, where as we already know $\mathbf{r}_{i}$ is the equilibrium one, $\Delta \mathbf{r}_{i, v b}$ is vibrational displacement around and relative to the equilibrium, and $\Delta \mathbf{r}_{i, t h}$ is thermal displacement around and relative to the vibration. In other words, it performs three motions at the same time: equilibrium postion motion (static once achieved), ordered vibration, and disordered thermal motion. Then the kinetic energy of the particles can be separated into two additive terms of ordered motion and disordered thermal motion respectively, and the ordered kinetic energy can be further separated into two additive ones of the DOF variables (but zero here) and of the vibration. Additionally kinetic energy of free electrons should also be considered, which are also "particles", while potential energy associated with electrons is always included in the total potential energy in any form. Then Eq.(8) can be further written as

$$
\tau=\tau_{v b}+\tau_{t h}+\tau_{e l},
$$

where $\tau_{e l}, \tau_{t h}$, and $\tau_{v b}$ are contribued from the kinetic energies of free electron's motion, (other) particle's thermal motion (disordered), and particle's vibrations (ordered) respectively. Specifically,

$$
\begin{aligned}
\tau_{v b} & =\frac{2}{3 V} E_{k, M D, v b} \mathbf{I}, \\
\tau_{e l} & =\frac{2}{3 V} E_{k, M D, e l} \mathbf{I},
\end{aligned}
$$

where $E_{k, M D, v b}$ and $E_{k, M D, e l}$ are the kinetic energies of the particle's vibrations and free electron's motion per cell. For the particle's thermal motion, it could be written as

$$
\tau_{t h}=\frac{1}{V} n k T \mathbf{I}
$$


where $k$ is Boltzmann constant and $T$ is the temperature. However any of these tensor items may be ignored if the corresponding actual physics process is negligible.

The second tensor term of the internal stress is

$$
\varepsilon=-\frac{1}{V} \sum_{\mathbf{z} \in \mathrm{DOF}}\left(\frac{\partial E_{p, M D}}{\partial \mathbf{z}}\right) \otimes \mathbf{z}
$$

where $E_{p, M D}$, a function of the DOF, is the total potential energy of a cell, which should be the same as $E$ in Eq.(1). Explicitly, it is

$$
\varepsilon=\frac{1}{V}\left(\sum_{i=1}^{n} \mathbf{F}_{i} \otimes \mathbf{r}_{i}-\frac{\partial E}{\partial \mathbf{a}} \otimes \mathbf{a}-\frac{\partial E}{\partial \mathbf{b}} \otimes \mathbf{b}-\frac{\partial E}{\partial \mathbf{c}} \otimes \mathbf{c}\right) .
$$

Considering Eq.(3) (equilibrium states), it becomes

$$
\varepsilon=-\frac{1}{V}\left(\frac{\partial E}{\partial \mathbf{a}} \otimes \mathbf{a}+\frac{\partial E}{\partial \mathbf{b}} \otimes \mathbf{b}+\frac{\partial E}{\partial \mathbf{c}} \otimes \mathbf{c}\right) .
$$

Since in equilibrium states $\ddot{\mathbf{h}}=0$ and using $\mathbf{h}^{\prime} \cdot \sigma_{h}=\delta_{\mathbf{h}, \mathbf{h}^{\prime}} V$, where $\mathbf{h}^{\prime}=\mathbf{a}$ or $\mathbf{b}$ or $\mathbf{c}$, Eq.(6) is

$$
\tau \cdot \sigma_{h}-\frac{\partial E}{\partial \mathbf{h}}+\mathbf{\Upsilon} \cdot \sigma_{h}=0 \quad(\mathbf{h}=\mathbf{a}, \mathbf{b}, \mathbf{c}) .
$$

As shown in Fig. 1 of our paper[20], if the crystal is imagined being cut into two halves, the terms $\mathbf{\Upsilon} \cdot \sigma_{h},-\partial E / \partial \mathbf{h}$, and $\tau \cdot \sigma_{h}$ in Eq.(16) are the exteranl force acting on the right end surface of, the net force by the whole left half of the crystal acting on, and the total force associated with momentum transportation acting on the "half-cell bar" $B_{\mathbf{h}}$ respectively. Since the net of all forces by other particles in the right half of the crystal on the "half-cell bar" $B_{\mathbf{h}}$ is zero, Eq.(16) means that the net of all internal and external forces on the "half-cell bar" $B_{\mathbf{h}}$ is zero, and then on each half of the crystal is also zero. Then the combination of Eq.(3) and Eq.(16) determine the crystal structure for given external temperature and stress. For equilibrium states, the internal temperature should be the same as the external temperature. As the period vectors determine the cell volume, they are actually also equations of state of crystals, but in a microscopic form.

\section{EQUATIONS OF STATE UNDER EXTERNAL PRESSURE}

For the special case of external pressure $P, \boldsymbol{\Upsilon}=-P \mathbf{I}$ (real number $P$ shoule be positive for compressing and negative for stretching), Eq.(16) becomes

$$
\tau \cdot \sigma_{h}-\frac{\partial E}{\partial \mathbf{h}}-P \sigma_{h}=0 \quad(\mathbf{h}=\mathbf{a}, \mathbf{b}, \mathbf{c})
$$

Comparing Eq.(4) and Eq.(17), we have the extra kinetic energy term reflecting the influence of temperature on crystal structures.

Supposing Eq.(17) is satisfied (equilibrium states), by dot mulitiplying it with period vector h, we can get the equations of state under external pressure in the following explicit form

$$
n k T+\frac{2}{3} E_{k, M D, e l}+\frac{2}{3} E_{k, M D, v b}+W_{i n v}-P V=0
$$

where the invariant work is

$$
W_{i n v}=-\mathbf{a} \cdot \frac{\partial E}{\partial \mathbf{a}}=-\mathbf{b} \cdot \frac{\partial E}{\partial \mathbf{b}}=-\mathbf{c} \cdot \frac{\partial E}{\partial \mathbf{c}} .
$$

Eq.(18) also allows us to specify the internal pressure as

$$
P_{i n t}=-\frac{2}{3 V} E_{k, M D}-\frac{W_{i n v}}{V},
$$

then

$$
P_{\text {int }}+P=0
$$


Eq.(18) and Eq.(20) are actually both equations of state, depending on external or internal pressure employed, for crystals under external pressure. Since they were derived based on the fact that the net of all internal and external forces on the above mentioned "half-cell bar" $B_{\mathbf{h}}$ is zero, they rigorously reflect the equilibrium condition of the crystal, from the perspective of forces.

If no free electrons, no (collective) vibrations, and no interactions between any particles (then no potential energer), Eq.(18) becomes

$$
P V=n k T
$$

which is the equation of state for ideal gases (in a cell of crystal). It can be re-written as $P \sigma_{h}=(n k T / V) \sigma_{h} \quad(\mathbf{h}=$ $\mathbf{a}, \mathbf{b}, \mathbf{c})$, where the left term $P \sigma_{h}$ and the right term $(n k T / V) \sigma_{h}$ are the net external force and the net internal force associated with momentum transportation acting on the "half-cell bar" $B_{\mathbf{h}}$ of cells filled with such ideal gases respectively. Then Eq.(22) is also the equilibrium condition of the ideal gases, from mechanical point of view. Additionally, the period vectors can be of any values, as long as satisfying $V=\sigma_{a} \cdot \mathbf{a}$. $[16]$

Now let us compare Eq.(18) with the well know Mie-Gruneisen equation, equation (4.46) of Born and Huang's book

$$
P=-\frac{d E}{d V}+\gamma \frac{E_{t, M D, v b}}{V}
$$

where $E_{t, M D, v b}$ is total energy of phonons of a cell and Gruneisen constant $\gamma$ is defined as

$$
\gamma=-\frac{d \ln \omega}{d \ln V}
$$

with respect to phonon frequency $\omega$. For simplicity, let us consider the cell in the shape of a rectangular prism, then in Cartesian coordinates, the period vectors can be written as $\mathbf{a}=(a>0,0,0), \mathbf{b}=(0, b>0,0)$, and $\mathbf{c}=(0,0, c>0)$. The derivative in Eq.(23) means

$$
\begin{aligned}
-\frac{d E}{d V}= & \lim _{\delta a, \delta b, \delta c \rightarrow 0}-\frac{E(a+\delta a, b+\delta b, c+\delta c)-E(a, b, c)}{b c \delta a+a c \delta b+a b \delta c} \\
= & \lim _{\delta a, \delta b, \delta c \rightarrow 0}-\frac{E(a+\delta a, b+\delta b, c+\delta c)-E(a, b+\delta b, c+\delta c)}{b c \delta a+a c \delta b+a b \delta c}+ \\
& +\lim _{\delta a, \delta b, \delta c \rightarrow 0}-\frac{E(a, b+\delta b, c+\delta c)-E(a, b, c+\delta c)}{b c \delta a+a c \delta b+a b \delta c}+ \\
& +\lim _{\delta a, \delta b, \delta c \rightarrow 0}-\frac{E(a, b, c+\delta c)-E(a, b, c)}{b c \delta a+a c \delta b+a b \delta c} \\
= & \lim _{\delta a, \delta b, \delta c \rightarrow 0}-\frac{E(a+\delta a, b, c)-E(a, b, c)+E(a, b+\delta b, c)-E(a, b, c)+E(a, b, c+\delta c)-E(a, b, c)}{b c \delta a+a c \delta b+a b \delta c}
\end{aligned}
$$

On the other hand, Eq.(19) can be written as

$$
\frac{W_{i n v}}{V}=\lim _{\delta a \rightarrow 0}-\frac{E(a+\delta a, b, c)-E(a, b, c)}{b c \delta a}=\lim _{\delta b \rightarrow 0}-\frac{E(a, b+\delta b, c)-E(a, b, c)}{a c \delta b}=\lim _{\delta c \rightarrow 0}-\frac{E(a, b, c+\delta c)-E(a, b, c)}{a b \delta c} .
$$

We arrived at

$$
-\frac{d E}{d V}=\frac{W_{i n v}}{V}
$$

Ignoring the free electron kinetic energy and the disordered thermal kinetic energy and considering the total kinetical energy of phonons in the cell being half of the total energy of phonons in the cell, Eq.(18) is the same as Mie-Gruneisen equation, Eq.(23), if Gruneisen constant is assumed

$$
\gamma=\frac{1}{3}
$$

Recalling Eq.(24), Eq.(28) means phonon frequency

$$
\omega \propto V^{-1 / 3}
$$

where $V^{-1 / 3}$ is actually the reciprocal of the one-dimentional length of the cell. Eq.(29) is actually shown as in equations (17) and (21) in chapter 5 of Kittel's book [15]. 


\section{THERMODYNAMICS}

Since crystal structures can be determined based on the microscopic equations of state (Eq.(3) and Eq.(16)), let us explore their thermodynamical properties and processes in the following three cases.

\section{A. Isovolumic}

Now let us consider the temperature is changed from $T$ to $T^{\prime}$ but keep the volume fixed. Actually, the fixed volume means the crystal structure does not change, then Eq.(3) is always satisfied, and the total potential energy is also fixed. However, in order to satisfy Eq.(16), since $\partial E / \partial \mathbf{h}$ does not change, the externally applied stress must change accordingly from $\Upsilon$ to $\Upsilon^{\prime}$ as the temperature dependent (kinetic energy) part of the internal stress changes from $\tau$ to $\tau^{\prime}$

$$
\mathbf{\Upsilon}^{\prime}=\mathbf{\Upsilon}+\tau-\tau^{\prime}
$$

The heat capacity at constant volume is then

$$
C_{V}=\left(\frac{d E_{k, M D}}{d T}\right)_{V}
$$

\section{B. Isobaric}

When the temperature is changed from $T$ to $T^{\prime}$, even with the external stress $\Upsilon$ kept fixed, the crystal structure must change accordingly in order to satisfy Eq.(3) and Eq.(16). As a result, the volume should change from $V$ to $V^{\prime}$, for an example. Then the coefficient of isobaric thermal expansion can be quickly calculated as

$$
\zeta=\frac{1}{V}\left(\frac{d V}{d T}\right)_{\Upsilon} \approx \frac{1}{V} \frac{V^{\prime}-V}{T^{\prime}-T} .
$$

The heat capacity at constant external pressure

$$
C_{P}=\left(\frac{d\left(E_{k, M D}+E_{p, M D}\right)}{d T}+P \frac{d V}{d T}\right)_{P}
$$

can also be calucalated the same way.

An alternative more accurate way to calculate them is to take derivatives of Eq.(3) and Eq.(16) with respect to temperature, since the equations should always be satisfied even if the external conditions are changed:

$$
\begin{aligned}
& \frac{d \mathbf{F}_{i}}{d T}=0 \quad(i=1,2, \cdots, n), \\
& \frac{d}{d T}\left\{\tau \cdot \sigma_{h}-\frac{\partial E}{\partial \mathbf{h}}+\mathbf{\Upsilon} \cdot \sigma_{h}\right\}=0 \quad(\mathbf{h}=\mathbf{a}, \mathbf{b}, \mathbf{c}) .
\end{aligned}
$$

Please note that all these are vector equations, then they are total $3(n+3)$ scalar equations. In Cartesian coordinates, for every coordinate component $k=x, y, z$, the component $F_{i, k}$ of the force $\mathbf{F}_{i}$ is a function of DOF, then

$$
\begin{aligned}
\frac{d F_{i, k}}{d T}= & \sum_{\mathbf{z} \in \text { DOF }} \frac{\partial F_{i, k}}{\partial \mathbf{z}} \cdot \frac{d \mathbf{z}}{d T}=0 \\
& (i=1,2, \cdots, n),(k=x, y, z) .
\end{aligned}
$$

As all the partial derivatives $\partial F_{i, k} / \partial \mathbf{z}$ are supposed known, all these total $3 n$ equations are linear ones about the derivatives of DOF with respect to temperature $\left(d \mathbf{r}_{1} / d T, d \mathbf{r}_{2} / d T, \cdots, d \mathbf{r}_{n} / d T, d \mathbf{a} / d T, d \mathbf{b} / d T\right.$, and $\left.d \mathbf{c} / d T\right)$.

Very similarly,

$$
-\frac{d}{d T} \frac{\partial E}{\partial \mathbf{h}} \quad(\mathbf{h}=\mathbf{a}, \mathbf{b}, \mathbf{c})
$$


are all linear combinations of the derivatives of DOF with respect to temperature. For example, $\mathbf{h}=\mathbf{a}$, considering constant stress $\Upsilon$, then

$$
\frac{d}{d T}\left(\mathbf{\Upsilon} \cdot \sigma_{a}\right)=\mathbf{\Upsilon} \cdot \frac{d \sigma_{a}}{d T}=\mathbf{\Upsilon} \cdot\left(\frac{d \mathbf{b}}{d T} \times \mathbf{c}+\mathbf{b} \times \frac{d \mathbf{c}}{d T}\right),
$$

also a linear combination of the derivatives of DOF with respect to temperature. Assuming $d\left(\tau \cdot \sigma_{h}\right) / d T$ can also be expanded as a linear combination of the derivatives of DOF with respect to temperature, we will get a system of $3(n+3)$ linear equations about the derivatives of DOF with respect to temperature. Once solved, the coefficients of isobaric thermal expansion can be calculated as

$$
\zeta=\frac{1}{V}\left(\frac{d V}{d T}\right)_{\mathbf{\Upsilon}}=\sum_{\mathbf{h}=\mathbf{a}, \mathbf{b}, \mathbf{c}} \frac{\sigma_{h}}{V} \cdot \frac{d \mathbf{h}}{d T} .
$$

The heat capacity at constant external pressure can be calculated the same way.

If the system expansion is assumed/proven evenly to certain degrees, the number of independent equations of the linear system should be reduced significantly.

\section{Isothermal}

In this case, an external pressure, rather than a stress, is applied to the system. Everything is similar to the isobaric case, but the derivatives shoule be taken of Eq.(3) and Eq.(18) with respect to pressure:

$$
\begin{aligned}
& \frac{d \mathbf{F}_{i}}{d P}=0 \quad(i=1,2, \cdots, n), \\
& \frac{d}{d P}\left\{\frac{2}{3} E_{k, M D, e l}+\frac{2}{3} E_{k, M D, v b}+W_{i n v}-P V\right\}=0 \quad(\mathbf{h}=\mathbf{a}, \mathbf{b}, \mathbf{c}) .
\end{aligned}
$$

Considering $d T / d P=0$ for isothermal condition and supposing the above equations also generate a system of $3(n+3)$ linear equations but about the derivatives of DOF with respect to pressure, the coefficients of isothermal compressibility can be calculated as

$$
\beta=-\frac{1}{V}\left(\frac{d V}{d P}\right)_{T}=-\sum_{\mathbf{h}=\mathbf{a}, \mathbf{b}, \mathbf{c}} \frac{\sigma_{h}}{V} \cdot \frac{d \mathbf{h}}{d P} .
$$

\section{PHONON EFFECTS}

Since many studies show that phonons play a very important role in crystal thermodynamical processes[8-14], let us consider phonons. Based on quantum statistics, the total energy of phonons of fixed frequency $\omega_{j}$ is $[11,15-19]$

$$
E_{t, j}=\frac{1}{2} \hbar \omega_{j}+\frac{\hbar \omega_{j}}{\exp \left(\hbar \omega_{j} / k T\right)-1}
$$

where $\hbar=h / 2 \pi$ and $h$ is Planck's constant. Then the total potential energy of phonons per cell is

$$
E_{t, M D, v b}=\sum_{j=1}^{3 n} E_{t, j}
$$

As a matter of fact, the kinetic energy and potential energy is averagely equal in harmonic vibrations, then the total kinetic energy of phonons per cell should be

$$
E_{k, M D, v b}=\frac{1}{2} E_{t, M D, v b}=\frac{1}{2} \sum_{j=1}^{3 n} \hbar \omega_{j}\left(\frac{1}{2}+\frac{1}{\exp \left(\hbar \omega_{j} / k T\right)-1}\right)
$$

The other half is the total potential energy of phonons per cell $E_{p, M D, v b}=\frac{1}{2} E_{t, M D, v b}$. Theoretically it should be included in $E$, the total potential energy of a cell, in Eq.(16). As in our paper[20], the term $-\partial E / \partial \mathbf{h}$ in Eq.(16) 
means the net force on the "half-cell bar" $B_{\mathbf{h}}$ in the right half of the crystal by all particles in the left half of the crystal. For any single force of such on a particle, which is vibrating around the equilibrium position as a harmonic oscillator, although the vibration causes the force changing a little bit constantly, the force should be averagely the one in the equilibrium state without vibration, as the force change is proportional to the relative displacement of the particle to its equilibrium position. This means that the averaged change caused by vibrational motion on the forces acting on the "half-cell bar" $B_{\mathbf{h}}$ by the left half of crystal, $-\partial\left(E_{p, M D, v b}\right) / \partial \mathbf{h}$, is zero, then the phonon's potential energy $E_{p, M D, v b}$ can be ignored in Eq.(16). Actually, neither kinetic energy nor potential energy of phonons affects Eq.(3). However both of them shoule be reflected in heat capacity calculation.

Bringing Eq.(45) into Eq.(10) then into Eq.(16) (and Eq.(6)), regardless of other kinetic energy, it shows that the period vectors should change if the temperature is changed while keeping the external stress fixed. This thermal expansion by pure harmonic vibrating phonons is contrary to usual ideas.

The calculation should be feasible by employing Debye-Slater model[10] for an example, where the total energy of phonons is

$$
E_{t, M D, v b}=\frac{9}{8} n k \Theta_{D}+3 n k T D\left(\frac{\Theta_{D}}{T}\right),
$$

where $\Theta_{D}$ is Debye temperature and function $D(\cdots)$ is Debye integral

$$
D(x)=\frac{3}{x^{3}} \int_{0}^{x} \frac{y^{3}}{e^{y}-1} d y .
$$

Then the total kinetic energy of phonons per cell $E_{k, M D, v b}=\frac{1}{2} E_{t, M D, v b}$ can be brought into Eq.(16).

\section{SYSTEM COLLAPSE}

Careful inspection on Eq.(16) (also based on Eq.(6)) shows that actually the temperature effect, the first term on the left side of Eq.(16), is to try to "break" the crystals, i.e. playing a repulsive role inside the system. Then particles inside the system should attract each other enough to overcome it in order to keep crystal stable. However, there is a limit to the overall attraction inside the crystal (the middle term of the left side of Eq.(16)). For fixed external stress, if the temperature is increased so much that its repulsive effect is bigger than the internal attraction limit and the external stress compensation, the crystal will collapse, where Eq.(16) can no longer be satisfied. On the other hand, for whatever temperature, if the external stress is stretching and bigger than the internal attraction limit, the system will also collapse. The higher temperature, the less stretching is needed to break the crystal system.

\section{SUMMARY}

By employing Newton's second law on particles in a cell and applying the dynamical equations of period vectors, crystal structures can be predicted. Not only the action of the external stress/pressure is presented in these equations, but also that of the (external) temperature via the kinetic energy term of the internal stress. They are actually equations of state of crystals in a microscopic form, and also the equilibrium conditions from the perspective of forces. For external pressure, they became Mie-Gruneisen equation supposing the Gruneisen constant is $1 / 3$, which means phonon frequency is inversely proportional to the cell length. Then thermodynamical properties and processes can be calculated as well. Contrary to usual ideas, the equations show that pure harmonic vibrating phonons can explain thermal expansion in crystals when temperature is changed. Furthermore crystals are also shown to collapse when the equations can not be satisfied.

\section{Acknowledgments}

The author wishes to thank Prof./Dr. Jonas Nyman, Univ Wisconsin, Madison, Dennis M. Elking, Openeye Sci Software, Santa Fe, JianDong Xu, Johnson \& Johnson Vision, Milpitas, YanBin Ji, Keck School of Medicine, University of Southern California, Los Angeles, USA, ShanQi Liu, Sun Yat Sen Univ, Sch Eah Sci \& Engn, Guangzhou, YongBing Li, Univ Chinese Acad Sci, Key Lab Computat Geodynam, Beijing, DianHong Shen, Institute of Physics, Beijing, CAS, JianZhong Zhang, School of Physics, Jilin Univerisity, Changchun, DanShu Yao, Department of Chemistry, College of Science, Northeastern University, Shenyang, Jin Huang, Shenyang No. 202 Hospital, Yan Xiao, College of 
Physics and Electronics Engineering, Shanxi University, Taiyuan, YongChao Xing, Shenyang Machine Tool (Group) Co., LTD, and XiaoDong Li, Institute of Metal Research, Shenyang, CAS, China, for their helpful discussions and criticisms.

[1] A. Gavezzotti, Acc. Chem. Res. 27, 309 (1994), doi:10.1021/ar00046a004.

[2] J. D. Dunitz, CHEM. COMMUN., 545 (2003), doi:10.1039/B211531J.

[3] C. W. Lehmann, Angew. Chem. Int. Ed. 50, 5616 (2011), doi:10.1002/anie.201101530.

[4] G.M. Day To cite this article: Graeme M. Day, Crystallography Reviews, 17 (2011) 3, doi: 10.1080/0889311X.2010.517526

[5] D.M. Elking, L. Fusti-Molnar, A. Nichols, Acta Cryst. B72, 488 (2016), doi:10.1107/S2052520616010118.

[6] J. Nyman, S.M. Reutzel-Edens, Faraday Discussions, 211, 459 (2018), doi:10.1039/c8fd00033f.

[7] G. Liu, E.G. Wang, and D.S. Wang, Chin. Phys. Lett. 14, 764 (1997), doi:10.1088/0256-307X/14/10/012.

[8] M.A. Blanco, E. Francisco, V. Luana, Comput. Phys. Commun. 158 (2004) 57, doi: 10.1016/j.comphy.2003.12.001.

[9] A. Otero-de-la-Roza, V. Luana, Comput. Phys. Commun. 182 (2011) 1708, doi:10.1016/j.cpc.2011.04.016.

[10] A. Otero-de-la-Roza, D. Abbasi-Perez, V. Luana, Comput. Phys. Commun. 182 (2011) 2232, doi: 10.1016/j.cpc.2011.05.009.

[11] R.M. Wentzcovitch, Y.G. Yu, Z. Wu, Theoretical and Computational Methods in Mineral Physics: Geophysical Applications, 71, 59 (2010), doi: 10.2138/rmg.2010.71.4.

[12] S. Shang, Y. Wang, D. Kim, Z. Liu, Computational Materials Science, $47 \quad 1040$ (2010), doi: 10.1016/j.commatsci.2009.12.006.

[13] S. Liu, Y. Li, Y. Shi, Acta Petrologica Sinica, 34, 1813 (2018).

[14] K. Wang, S. Shang, Y. Wang, Z. Liu, F. Liu, Acta Materialia, 147, 261 (2018), doi:10.1016/j.actamat.2018.01.013.

[15] C. Kittel, Introduction to Solid State Physics (Sixth Edition), John Wiley \& Sons, New York. 1986.

[16] M. Born, K. Huang, Dynamical Theory of Crystal Lattices, Oxford University Press, London, 1954.

[17] D.S. Schmool, Solid State Physics from the Material Properties of Solids to Nanotechnologies, Mercury Learning and Information, Boston, 2017.

[18] O.L. Anderson, Equations of State of Solids for Geophysics and Ceramic Science, Oxford university press, New York, 1995

[19] Z.Z. Li, Solid State Theory, Higher Education Press, Beijing, 1985

[20] G. Liu, Can. J. Phys. 93, 974 (2015), doi:10.1139/cjp-2014-0518, arXiv:cond-mat/0209372 (version 16). 